\title{
ANÁLISE DO PADRÃO ESTACIONAL E PREVISÃO DOS PREÇOS DA SOJA AOS PRODUTORES DO ESTADO DE SÃO PAULO, BRASIL
}

\author{
Celso Correia de Souza ${ }^{1}$ \\ Janaína Outeiro de Andrade ${ }^{2}$ \\ Luiz Henrique Brito Lemes ${ }^{3}$ \\ Hevelyne Henn da Gama Viganó ${ }^{4}$ \\ Anderson Susumu Kazama ${ }^{5}$
}

\section{RESUMO}

Este artigo discute a sazonalidade dos preços da soja para os produtores de janeiro de 2000 a dezembro de 2016, no estado de São Paulo (SP). Considerando o preço como a variável mais importante na decisão de comercialização dessa commodity, foi analisado o índice de variação sazonal percentual (IVEP) da soja comercializada no estado de São Paulo, Brasil, bem como os limites inferior e superior de confiança percentuais (LICP e LSCP), no período de 2000 a 2016. Utilizando conceitos de séries temporais e da técnica ARIMA, analisou-se a tendência de preços da soja no período considerado e realizou-se uma provisão dos preços desse produto para os doze meses seguintes, ano de 2017. Os resultados constataram que os preços do saco de soja variaram muito, tanto dentro de um mesmo ano, quanto ao longo do período estudado (de 2000 a 2016). Em virtude da oferta de soja ser maior no primeiro semestre do ano devido a colheita do produto, os preços do saco de soja são menores nesse período e se elevam durante o segundo semestre. No período de março a maio de cada ano, apesar do preço praticado pelo mercado ser menor, o histórico dos preços mostra que o produtor pode vender o seu produto por um excelente preço, sem muitos riscos de prejuízos. As previsões dos preços do saco de soja para os meses do ano de 2017, logo após o período estudado, utilizando o modelo ARIMA se mostrou robusto, apresentando um bom desempenho, com pequenos erros de previsão.

Palavras-chave: IVEP do preço da soja, LICP e LSCP do preço da soja, mercado da soja; produção de soja.

\section{ANALYSIS OF THE SEASONAL PATTERN AND FORECAST OF SOYBEAN PRICES TO PRODUCERS IN THE STATE OF SÃO PAULO, BRAZIL}

\footnotetext{
ABSTRACT

This article discusses the seasonality of the prices of soybeans to producers, from January 2000 to December 2016, in the state of São Paulo (SP). Considering price as

${ }^{1}$ Graduado em Matemática. Mestrado em Matemática Aplicada. Doutorado em Engenharia Elétrica. Professor do Mestrado em Produção e Gestão Agroindustrial da Universidade Anhanguera Uniderp. E-mail: csouza939@gmail.com

${ }^{2}$ Graduada em Administração. Mestrado em Produção e Gestão Agroindustrial da Universidade Anhanguera Uniderp. Email: jan_andrade.adm@hotmail.com.

${ }^{3}$ Graduando em Agronomia pela Universidade Anhanguera Uniderp. E-mail: luizhblemes@gmail.com.

${ }^{4}$ Graduada em Física. Mestrado em Física. Doutorado em Meio Ambiente e Desenvolvimento Regional. Email:hevelyne.vigano@ifms.edu.br.

${ }^{5}$ Graduado em Administração. Mestrado em Produção e Gestão Agroindustrial. E-mail: a1011a@ymail.com.
} 
the most important variable in commercialization decision of this commodity, the seasonal variation index (IVEP) of soybean marketed in state of São Paulo, Brazil was analyzed, as well as the lower and upper confidence limits (LICP and LSCP), in period of 2000 to 2016. Using time-series concepts and the ARIMA technique, the soybean price trend was analyzed during the period considered with a prices provision of that product was made for the following twelve months in 2017 . The results showed that the prices of the bag of soybean varied greatly, both within the same year and over long the period studied (from 2000 to 2016). Due to the higher soybean supply in the first half of each year due to the harvest of the product, the soybean prices are lower during this period and increase during the second semester. In the period from March to May of each year, although the price practiced by the market is lower, historical prices show that the producer can sell his product at a great price, without many risks of losses. The forecast of soybean bag prices for the months of 2017, shortly after the period studied, using the ARIMA model was robust, showing a good performance, with small forecast errors.

Keywords: IVEP of the price of soy, LICP and LSCP of the price of soy, soybean market; soy production.

\section{INTRODUÇÃO}

O cultivo da soja é a atividade, dentro do agronegócio nacional, que apresenta maior expansão. Fatores como o desenvolvimento e a estruturação do mercado relacionado com o comércio de produtos do complexo soja, consolida essa oleaginosa como uma das mais representativas commodities dentro do agronegócio brasileiro. Por outro lado, as tecnologias desenvolvidas na melhoria da produção da soja, faz dessa oleaginosa a mais cultivada no Brasil. Além do mais, a soja é também uma commodity padronizada e uniforme, facilitando a sua produção e comercialização por produtores de diversos países.

No mercado brasileiro, segundo Paula e Faveret Filho (1998), a expansão da cultura da soja foi a principal responsável pela introdução do conceito de agronegócio no país, isso em função não só do aumento da área de produção, mas pelo incremento de produtividade, principalmente, nas áreas regiões Centro-Oeste e Sul do Brasil.

Segundo a Companhia Nacional de Abastecimento (CONAB), em 2015 o Brasil produziu 96,5 milhões de toneladas de soja, se tornando uns dos maiores produtores mundiais de soja, com Área plantada de 33,177 milhões de hectares. Com projeção de crescimento de $7,3 \%$ na produção, atingindo 102,4 milhões de toneladas para safra de 2016/2017 (CONAB, 2016).

Os principais produtores nacionais de soja são os estados de Mato Grosso, Paraná, Rio Grande do Sul, Goiás, Mato Grosso do Sul, Minas Gerais e São Paulo. Em 2015 houve um aumento na área plantada de soja no Brasil, particularmente, no estado de São Paulo, em virtude da expansão da soja sobre as áreas de cana-deaçúcar.

$\mathrm{Na}$ safra de 2015/2016 segundo o Instituto de Economia Agrícola (IEA), a safra cultivada de soja em SP teve um crescimento de $6,7 \%$, totalizando 808,7 mil hectares, com crescimento de $18,3 \%$ na produção, totalizando 2,64 milhões de toneladas do grão no Estado (IBGE, 2016; IEA, 2016).

A mecanização e as novas cultivares de soja, altamente produtivas e adaptadas às diferentes regiões do país, o desenvolvimento de pacotes tecnológicos relacionados ao manejo de solos, ao manejo de adubação e calagem, ao manejo de pragas e doenças, além de identificação e solução para os principais fatores 
responsáveis por perdas no processo de colheita, são responsáveis pelo crescimento da cultura da soja no país (FREITAS, 2011).

De uma forma geral, os preços dos produtos agropecuários são instáveis ao longo do tempo, por influência dos seguintes fatores: clima, pragas, dificuldades de previsão e, sazonalidade da produção de alimentos. Esses fatores dificultam o ajuste entre a produção e as nuances do mercado, o que obriga o produtor a ter maior cuidado com as oscilações de preços. Vários autores apontam para um comportamento volátil tanto na produção quanto do preço da soja, provocando inconstância ao produtor (BOTTINI et al., 1995; CAMARGO FILHO; MAZZEI, 2001; CRUZ et al., 2001).

O preço interno da soja é fundamentalmente determinado pelo preço internacional dessa commodity, cotado na Bolsa de Chicago, e pela taxa de câmbio real-dólar. Para Lovadine e Bacchi (2005), o Brasil é um grande produtor e exportador de óleo e farelos de soja, e esses produtos influenciam, de certa forma, o mercado nacional quando existe relação entre os seus preços internacionais e o preço da soja em grão.

O conhecimento por parte do produtor e do comerciante de soja da variação estacional dos preços é muito importante, para direcionar as decisões dos produtores agrícolas e dos comerciantes, bem como, para articulação de políticas agrícolas do governo. Desta forma, a análise da formação de preços no mercado agrícola é extremamente importante para orientar produtores e comerciantes nas tomadas de decisão de quanto produzir, ou de quanto comprar, com os objetivos de maximizar seus lucros, ou de minimizar os seus custos.

Diante do exposto, este estudo tem como objetivo compreender a variável preço no contexto do planejamento e nas tomadas de decisão na comercialização da soja no estado de São Paulo, através da análise de uma série histórica de preços do produto, no período de 2000 a 2016 que, de certa forma, reflete os preços da soja a nível nacional. Para a consecução desse objetivo se fez necessário determinar os limites de confiança inferior (LIC) e superior (LSC) dos preços da soja ao longo do ano, dos pontos de vista do produtor e do comerciante; eliminar os efeitos das sazonalidades dos preços da soja através da determinação das médias móveis dos preços desse produto.

\section{MATERIAL E MÉTODOS}

Inicialmente, foi feito um levantamento bibliográfico dos assuntos que envolviam o tema em estudo, em seguida, uma revisão bibliográfica desses assuntos para a fixação dos conceitos que foram utilizados. Esta pesquisa pode ser caracterizada com exploratória descritiva, pois, procurou-se, através de dados de preços da soja, pagos aos produtores, descobrir e observar como esses preços sofrem variações durante o ano, quais as tendências ao longo do tempo, procurando descrevê-las, classificá-las e interpretá-las.

Para a realização deste trabalho foram utilizados dados secundários dos preços pagos aos produtores na comercialização da soja, saco de $60 \mathrm{~kg}$, no estado de São Paulo, no período de janeiro de 2000 a dezembro de 2016, disponíveis no Instituto de Economia Agrícola (IEA).

Devido à constante desvalorização do real, motivada pela inflação, tornou-se necessário corrigir os preços do saco de soja no período analisado, com a determinação dos preços reais na moeda corrente. Usou-se a série histórica do Índice de Preço ao Consumidor Amplo (IPCA) no período, com correções referidas a 31 de dezembro de 2016. $\mathrm{Na}$ análise do comportamento e previsão do valor da soja, no período, foi usado o modelo Autorregressivo Integrado de Médias Móveis (ARIMA). 
A técnica ARIMA $(p, d, q)$ é um método sofisticado de Box-Jenkins para a análise de séries temporais que utiliza as correlações entre as observações dos dados em diversos instantes. Essa metodologia para a previsão se baseia no ajuste de modelos de séries temporais de valores observados de tal forma que as diferenças entre os valores gerados pelos modelos e os valores observados, resultem em séries de resíduos de comportamentos aleatórios em torno de zero (MORETTIN; TOLOI, 2006).

Os modelos ARIMA $(p, d, q)$ são "diferenciados" $d$ vezes da série original, possuem $p$ parâmetros autorregressivos e $q$ parâmetros de médias móveis. A ordem de diferenciação $d$ é o número de diferenças necessárias para tornar a série nãoestacionária em estacionária, removendo os efeitos de tendências e especifica a ordem de diferenciação da série antes de estimar os modelos. O termo autorregressivo $p$ especifica quais os valores anteriores da série serão utilizados para prever os valores atuais, e o número de ordens de médias móveis $q$ especifica como os desvios da média da série para valores anteriores são utilizados para prever os valores atuais (JACOBS; COSTA, 2011).

A forma genérica da modelagem é apresentada pela equação (1) (MORETTIN; TOLOI, 2006).

$$
(1-B)^{d} \phi(B) y_{t}=\theta(B) \varepsilon_{t}
$$

onde: $\phi(B)$ e $\theta(B)$ são os operadores autorregressivos e de médias móveis; $y_{t}$ é o valor de $y$ em determinado período de tempo $t$; $\varepsilon_{t}$ é o erro aleatório nãocorrelacionado, com média zero e variância constante (ruído branco); $B$ é o operador defasagem e $d$ é o número de diferenciações da série.

Para a execução desse método é necessário passar por quatro estágios principais: 1) identificação de modelos; 2) estimativa dos parâmetros; 3) análise dos resíduos e; 4 ) teste de adequação, aos quais se segue a aplicação do modelo para a previsão ou controle do sistema de geração dos valores observados, conforme a Figura 1 (JACOBS e COSTA, 2011). 
Figura 1 - Estágios da metodologia de Box-Jenkins (ARIMA).

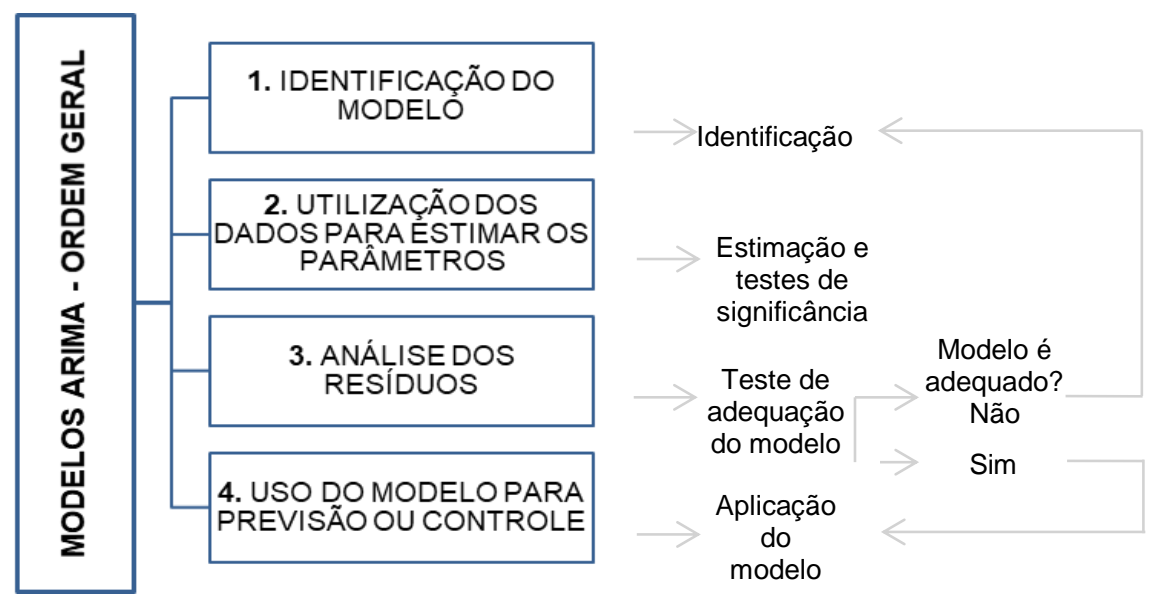

Fonte: Jacobs e Costa (2011).

O diagnóstico inicial para a verificação do modelo no que tange ao ajuste dos dados é a análise dos resíduos a partir das funções autocorrelação e autocorrelação parcial, que devem mostrar que os resíduos são puramente aleatórios, caso contrário, deve-se procurar por outro modelo ARIMA.

De acordo com Gujarati (2006), devem ser feitas as seguintes verificações no ajustamento de modelos ARIMA:

1) Verificar se a série é estacionária;

2) Examinar a matriz das correlações, onde não deve ocorrer um alto grau de correlação entre as estimativas dos parâmetros do modelo;

3) Verificar se todos os termos do modelo foram incluídos;

4) Examinar as estimativas dos parâmetros e seus desvios padrão, aplicando testes de hipóteses para avaliar a inclusão do parâmetro no modelo;

5) Verificar se a soma dos quadrados dos erros e o desvio padrão dos resíduos diminuem enquanto o ajuste do modelo melhora;

6) Observar se há padrão significante no correlograma dos resíduos e;

7) Analisar as combinações dos parâmetros a fim de evitar diferenças e parâmetros sazonais.

Feitas as verificações, deve-se então analisar as estatísticas do modelo a partir das informações de $\mathrm{R}^{2}$, raiz do erro quadrático médio (RMSE), erro percentual absoluto médio (MAPE), critério de informação Bayesiano (BIC) e a significância ( $p$ valor).

Nesse modelo, caso existam sazonalidades, ou seja, oscilações de curto prazo que ocorrem sempre dentro do ano e que se repetem sistematicamente ano após ano, deve-se aplicar as condições de sazonalidade para obter suas variações. Existem vários métodos para a obtenção das variações sazonais, bem como, os limites inferior e superior de variação no período, denominados de limites de confiança inferior e superior, respectivamente.

Segundo Arias et al. (2009), dentre os métodos mais utilizados, destaca-se o índice de variação estacional percentual (IVEP), definido pela equação (2). 


$$
I V E P=\frac{M m}{M g}
$$

Onde $M m=$ média aritmética de cada mês no intervalo considerado e; $M g=$ média geral dos valores da série no intervalo. Ainda, segundo Arias et al. (2009), os cálculos para os limites de confiança percentual inferior (LICP) e superior (LSCP) no intervalo são obtidos através das equações (3) e (4), respectivamente.

(3)

$$
L I C P=\frac{L}{M g}
$$

(4)

$$
L S C P=\frac{H}{M g}
$$

Onde $L=$ menor valor mensal da série no intervalo e, $H=$ maior valor mensal da série no intervalo.

\section{RESULTADOS E DISCUSSÃO}

Para que se pudessem fazer previsões sobre os preços do saco de soja em determinados instantes, tanto no período quanto exterior ao período considerado da série (2000 a 2016), foi realizada a correção dos preços pela série inflacionária do Índice de Preços ao Consumidor Amplo (IPCA) do período, e determinada a equação de ajustamento polinomial da série dos valores das médias móveis, bem como, o respectivo coeficiente de ajustamento $R^{2}$, para o estabelecimento da explicação do modelo e posteriormente aplicada a metodologia ARIMA.

Os valores da Tabela 1 retratam os preços corrigidos do saco de soja, comercializado no estado de São Paulo, referente ao período de janeiro de 2000 a dezembro de 2016.

\begin{tabular}{|c|c|c|c|c|c|c|c|c|c|c|c|c|}
\hline ANO & JAN & FEV & MAR & ABR & MAI & JUN & JUL & AGO & SET & OUT & NOV & DEZ \\
\hline 2000 & 54,41 & 55,90 & 50,47 & 50,31 & 51,88 & 49,82 & 47,25 & 45,82 & 49,15 & 49,03 & 50,12 & 53,94 \\
\hline 2001 & 53,09 & 48,27 & 47,79 & 46,96 & 46,87 & 51,71 & 59,89 & 62,27 & 65,45 & 71,09 & 70,92 & 65,59 \\
\hline 2002 & 62,08 & 55,97 & 50,66 & 50,25 & 53,09 & 60,61 & 69,92 & 77,01 & 83,87 & 96,81 & 101,28 & 103,15 \\
\hline 2003 & 99,39 & 92,82 & 87,21 & 80,87 & 76,82 & 76,89 & 77,38 & 75,24 & 80,80 & 89,72 & 99,52 & 96,02 \\
\hline 2004 & 98,29 & 94,68 & 101,72 & 106,39 & 104,06 & 90,16 & 85,72 & 76,06 & 78,86 & 71,36 & 68,16 & 66,13 \\
\hline 2005 & 63,19 & 59,21 & 65,56 & 63,46 & 58,99 & 60,51 & 60,37 & 59,65 & 56,55 & 54,28 & 52,82 & 53,23 \\
\hline 2006 & 54,02 & 52,32 & 48,88 & 45,62 & 44,98 & 47,69 & 47,08 & 47,00 & 48,08 & 50,31 & 53,72 & 54,47 \\
\hline 2007 & 53,82 & 57,04 & 56,09 & 52,40 & 51,47 & 52,37 & 54,04 & 55,98 & 60,52 & 65,45 & 68,35 & 70,70 \\
\hline 2008 & 73,04 & 77,23 & 78,78 & 75,19 & 76,02 & 80,80 & 84,09 & 72,75 & 73,88 & 72,51 & 71,46 & 70,52 \\
\hline 2009 & 74,22 & 76,13 & 72,64 & 73,78 & 77,66 & 77,70 & 76,37 & 74,74 & 72,52 & 73,26 & 71,91 & 71,15 \\
\hline 2010 & 64,28 & 55,88 & 53,41 & 52,83 & 55,30 & 55,46 & 56,36 & 62,69 & 64,55 & 67,64 & 71,35 & 73,43 \\
\hline 2011 & 73,64 & 73,58 & 68,44 & 65,53 & 64,17 & 65,47 & 63,92 & 65,19 & 68,36 & 66,17 & 64,41 & 62,24 \\
\hline 2012 & 63,77 & 64,25 & 69,16 & 75,33 & 80,38 & 83,23 & 92,15 & 107,17 & 115,08 & 108,72 & 96,48 & 97,16 \\
\hline 2013 & 85,75 & 78,27 & 75,65 & 71,62 & 72,80 & 83,35 & 82,82 & 81,13 & 86,27 & 84,84 & 87,52 & 85,21 \\
\hline 2014 & 78,47 & 76,94 & 76,93 & 77,49 & 74,32 & 72,93 & 68,07 & 64,87 & 65,43 & 62,24 & 66,60 & 67,45 \\
\hline 2015 & 63,93 & 61,65 & 66,65 & 69,02 & 66,01 & 65,54 & 67,11 & 72,04 & 72,49 & 76,62 & 78,42 & 78,22 \\
\hline 2016 & 75,84 & 72,42 & 70,91 & 69,08 & 76,98 & 87,56 & 79,08 & 76,37 & 73,47 & 72,07 & 69,48 & 70,85 \\
\hline $\mathbf{M}^{\star}$ & 70,07 & 67,80 & 67,12 & 66,24 & 66,58 & 68,34 & 68,92 & 69,18 & 71,49 & 72,48 & 73,09 & 72,91 \\
\hline $\mathrm{DP}^{*}$ & 13,98 & 13,37 & 14,40 & 15,15 & 14,93 & 13,79 & 13,25 & 13,85 & 15,24 & 15,28 & 14,91 & 14,48 \\
\hline
\end{tabular}

Tabela 1 - Preços corrigidos do saco de $60 \mathrm{~kg}$ de soja, comercializada no estado de São Paulo, no período de janeiro de 2000 à dezembro de 2016.

$\mathrm{M}^{*}$ : médias aritméticas mensais; $\mathrm{DP}^{\star}$ : desvios padrão mensais.

Fonte: Adaptado de IEA (2016) 
Pelos valores observados na Tabela 1 foi possível constatar que, de um modo geral, os meses de maiores variações nos preços médios do saco de soja é no período de março a maio de 2004, havendo uma queda nos meses de março a setembro de 2006, se mantendo estável nos anos subsequentes sem alterações bruscas nos preços até agosto de 2007.

Para Stefanelo (2008) a justificativa do aumento do preço do saco de soja no período de março a maio de 2004 foi em virtude da redução da safra 2003/04 americana e brasileira, as cotações se elevaram durante o segundo semestre de 2003 e durante o primeiro trimestre de 2004 (o segundo maior preço de todos os tempos). Após esse período, a safra americana recuperou-se e as cotações médias tiveram baixas e, nos meses seguintes, oscilações.

Nesse período era esperado que os Estados Unidos alcançassem os números das projeções divulgadas, porém, com estranheza que foi recebido a divulgação da queda de produtividade em meio a um quadro favorável, logo após essa a divulgação, o Brasil registrou alta nos preços do saco da soja.

Para Fürstenau (2004), aumento dos preços de venda do grão de soja no mercado internacional da safra de $2003 / 04$ foi decorrente das baixas na safra norte americana e do crescimento do consumo, especialmente na China. A combinação dessas variáveis gerou os menores estoques internacionais do grão desde 1976/77.

A produção nacional, na safra de $2003 / 2004$, foi de $49.792,7$ mil t e da safra de $2004 / 2005$ foi de $50.229,9$ mil t. A produção paulista de soja na safra de 2003/04 foi estimada em 1.828,0 mil t, aumenta $11,6 \%$ em relação ao ano anterior. A sojicultora no estado de São Paulo, a exemplo das demais regiões produtoras do Brasil, sofreu na safra adversidade climática (estiagem) e ocorrência de doenças (principalmente a ferrugem asiática), que afetaram negativamente o desenvolvimento vegetativo, a produção e a qualidade de grãos (TSUNECHIRO, 2004).

Já, em 2005, em decorrência da previsão inicial de uma safra recorde nos Estados Unidos, e expectativa de alta da safra da América Sul, os preços mantiveram-se baixos no primeiro semestre, porém, o Departamento de Agricultura dos Estados Unidos (USDA) divulgou em agosto de 2005 a primeira estimativa efetiva para a safra de soja norte-americana de 2005/06, com um volume abaixo do esperado. Apesar dessas informações, a soja no Brasil não teve uma alta como nos anos anteriores, mas com as margens econômicas negativas do algodão podem, em caso de continuidade, gerar substituição por soja, como por exemplo, no Mato Grosso. Já, na região Sul, a soja também tende a ganhar áreas e, para o estado de São Paulo, houve uma redução de $4,8 \%$ na área cultivada e de $2,3 \%$ na produção (AMARO et al., 2006).

Ainda, segundo a Tabela 1, houve um aumento dos preços a partir de dezembro de 2007, se mantendo estável até dezembro de 2009, demonstrando a sazonalidade no mercado. Pode-se notar que nos meses de fevereiro a julho de 2010 houve uma queda no preço do saco de soja comercializado, tendo uma recuperação no mês de agosto de 2010. A partir de julho de 2014 se observa novamente uma queda no valor comercializado do produto até o mês de fevereiro de 2015 , sendo que a partir de março constata-se a recuperação gradativa do valor comercializado.

Como observado pelos dados da Tabela 1, o preço da soja sofreu forte alta a partir de 2012, impulsionado pelos baixos estoques do grão no país e por uma demanda internacional aquecida. Desde que o grão rompeu a barreira dos $R \$ 70,00$ por saca em 2012, os patamares de preços da soja têm oscilado acima deste valor. A exemplo do milho, o efeito do câmbio torna mais atrativa a procura pela soja brasileira para exportação e, consequentemente, pressionando o preço da soja para cima. 
No período analisado nesta pesquisa (janeiro de 2000 a dezembro de 2016), foi observado decréscimos dos preços da soja nos anos de 2005 até outubro de 2006 (Tabela 1), havendo uma retomada com acréscimos nos preços comercializado no período de julho de 2015 a janeiro de 2016, indicando uma tendência de acréscimo nas exportações deste produto na região em estudo.

A Tabela 2 apresenta o índice de variação estacional percentual (IVEP), bem como os limites de confianças mensais percentuais, inferior (LICP) e superior (LSCP), ou seja, retrata, em percentagens, os limites inferior e superior de preços de cada mês obtidos pelo saco de soja de $60 \mathrm{~kg}$, comercializada no período analisado, podendo ser considerado como uma indicação do risco de mercado.

Tabela 2 - Índice de Variação Estacional IVEP, Limite Inferior de Confiança (LICP) e Limite Superior de Confiança (LSCP), do saco de soja no período de 2000 a 2016, no estado de São Paulo.

\begin{tabular}{cccc}
\hline \multirow{2}{*}{ Mês } & \multirow{2}{*}{ IVEP (\%) } & \multicolumn{2}{c}{ Limites de Confiança (\%) } \\
\cline { 3 - 4 } & & LICP & LSCP \\
\hline Jan & 100,79 & 76,37 & 142,96 \\
Fev & 97,52 & 69,44 & 136,19 \\
Mar & 96,54 & 68,74 & 146,32 \\
Abr & 95,29 & 65,62 & 153,04 \\
Mai & 95,77 & 64,71 & 149,69 \\
Jun & 98,30 & 68,60 & 129,70 \\
Jul & 99,13 & 67,72 & 132,55 \\
Ago & 99,50 & 65,92 & 154,16 \\
Set & 102,83 & 69,16 & 165,53 \\
Out & 104,26 & 70,52 & 156,39 \\
Nov & 105,13 & 72,09 & 145,68 \\
Dez & 104,88 & 76,57 & 148,37
\end{tabular}

Fonte: próprio autor.

O índice de variação estacional percentual (IVEP), mostrado na Tabela 2, evidencia que os meses com os maiores índices de preços foram no período de meados de agosto a dezembro. Na Figura 1 estão os gráficos dos três índices retratados na Tabela 2 (IVEP LICP e LSCP).

Figura 1 - Índices percentuais de variações estacionais IVEP e Limite Inferior e Superior de Confiança do preço da soja (LICP e LSCP) e desvio padrão do saco de $60 \mathrm{Kg}$ de soja, de 2000 à 2016, no estado de São Paulo. 


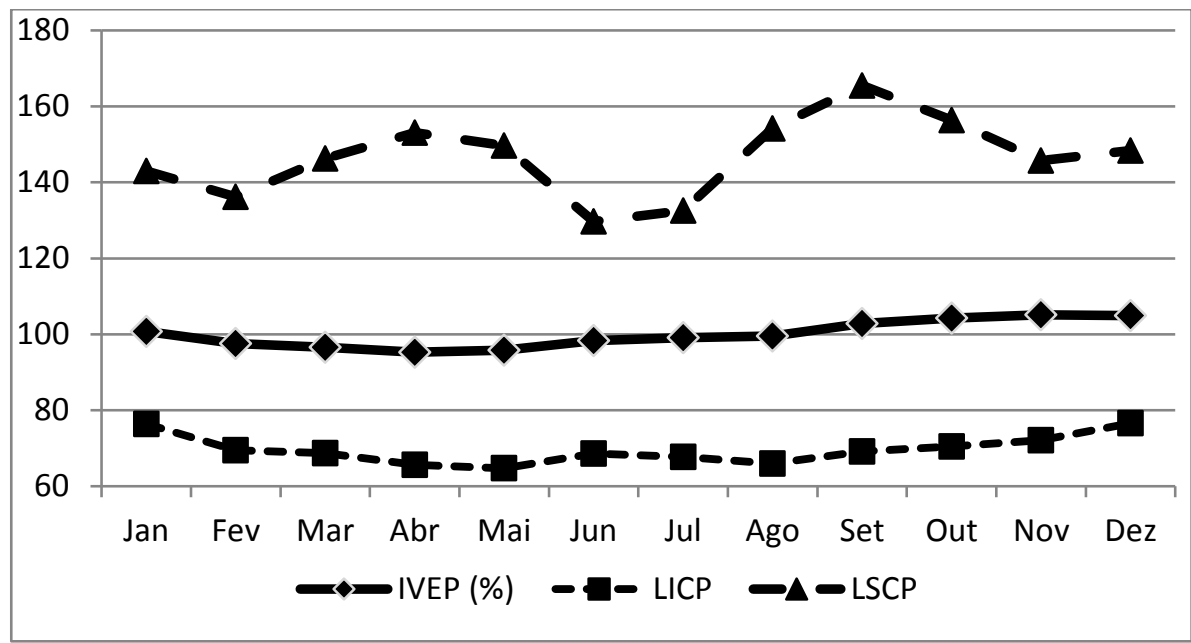

Fonte: próprio autor.

Desta forma, a análise dos valores retratados na Figura 1 permite concluir que os meses que apresentam menores riscos de comercialização para o produtor seriam aqueles que o valor do limite inferior menos se distancia da média histórica de preços. Isso quer dizer que, nos meses onde o limite inferior ficou mais próximo da média geral histórica são os que o produtor tem menores chances de perdas econômicas. Sendo assim, pode-se observar na Figura 1 que os meses com menores riscos (limite inferior), e maiores chances de ganhos (limite superior) para o produtor comercializar soja em julho $(69,17 \%$ a $126,35 \%)$, agosto $(66,90 \%$ a $118,23 \%)$, setembro $(70,41 \%$ a $124,94 \%)$ e outubro $(71,62 \%$ a $141,04 \%)$.

Já os valores dos limites inferior e superior de março (69,79\% a 148,85\%), abril $(67,20 \%$ a $155,97 \%)$ e maio $(66,56 \%$ a $152,77 \%)$ permitem inferir que, também nesses meses o produtor também pode obter grandes lucratividades na comercialização de soja, sem correr muito risco de algum prejuízo. Essa maior variação nos preços praticados nesses meses indicam uma maior variação no abastecimento de um ano para o outro.

Cruzando os dados apresentados nas Tabelas 1 e 2, pode-se observar que, de um modo geral, os meses nos quais ocorreram os maiores preços deste produto foram os que apresentam os menores volumes comercializados.

A Tabela 3 ilustra as previsões de preços obtidos pelo método ARIMA, do saco de soja comercializada no estado de São Paulo no período de janeiro de 2000 a dezembro de 2016.

Tabela 3 - Preços previstos pela metodologia $\operatorname{ARIMA}(0,1,5)$ do saco de soja, no período de 2000 a 2016, comercializado no estado de São Paulo.

\begin{tabular}{|c|c|c|c|c|c|c|c|c|c|c|c|c|}
\hline ANO & JAN & FEV & MAR & ABR & MAI & JUN & JUL & AGO & SET & OUT & NOV & DEZ \\
\hline 2000 & 54,50 & 56,49 & 48,48 & 51,13 & 52,05 & 48,97 & 47,56 & 44,95 & 50,88 & 48,77 & 51,08 & 55,57 \\
\hline 2001 & 51,42 & 47,47 & 47,77 & 46,28 & 47,57 & 54,14 & 62,37 & 62,53 & 66,61 & 72,00 & 69,37 & 64,28 \\
\hline 2002 & 60,84 & 53,63 & 49,75 & 51,04 & 54,35 & 64,33 & 73,12 & 78,61 & 85,60 & 99,88 & 100,53 & 103,44 \\
\hline 2003 & 96,89 & 89,45 & 86,28 & 78,61 & 76,80 & 77,65 & 77,76 & 75,25 & 83,59 & 92,41 & 102,73 & 94,20 \\
\hline 2004 & 98,88 & 92,07 & 104,62 & 108,58 & 101,74 & 86,77 & 83,97 & 73,05 & 82,04 & 69,11 & 68,05 & 66,65 \\
\hline 2005 & 61,15 & 60,02 & 68,17 & 62,11 & 58,45 & 61,80 & 58,99 & 60,78 & 55,56 & 53,55 & 52,85 & 53,37 \\
\hline 2006 & 55,07 & 51,57 & 48,05 & 44,71 & 45,07 & 49,30 & 46,75 & 47,62 & 48,30 & 50,71 & 55,51 & 54,11 \\
\hline 2007 & 53,71 & 58,10 & 54,85 & 51,72 & 51,51 & 52,25 & 55,20 & 56,85 & 62,20 & 66,70 & 68,72 & 71,45 \\
\hline 2008 & 73,00 & 78,40 & 78,73 & 73,59 & 76,84 & 81,75 & 85,11 & 69,05 & 75,58 & 70,82 & 71,48 & 72,24 \\
\hline 2009 & 74,26 & 77,59 & 70,78 & 75,31 & 78,37 & 77,23 & 77,06 & 73,43 & 71,90 & 74,05 & 71,35 & 71,58 \\
\hline 2010 & 61,85 & 53,60 & 53,71 & 52,61 & 57,59 & 55,73 & 56,75 & 65,51 & 63,72 & 69,84 & 71,94 & 72,87 \\
\hline
\end{tabular}




\begin{tabular}{|c|c|c|c|c|c|c|c|c|c|c|c|c|}
\hline 2011 & 74,27 & 72,67 & 66,70 & 64,95 & 63,86 & 66,35 & 63,77 & 66,08 & 69,55 & 64,71 & 64,83 & 61,11 \\
\hline 2012 & 64,58 & 64,80 & 71,15 & 77,75 & 80,98 & 84,36 & 94,50 & 111,7 & 115,96 & 105,65 & 91,79 & 97,36 \\
\hline 2013 & 81,26 & 78,14 & 76,03 & 69,37 & 76,02 & 87,17 & 81,77 & 81,88 & 87,48 & 82,61 & 90,51 & 83,46 \\
\hline 2014 & 76,01 & 77,87 & 75,92 & 79,10 & 73,43 & 72,70 & 66,58 & 64,09 & 66,82 & 60,70 & 70,02 & 66,88 \\
\hline 2015 & 62,68 & 62,13 & 67,53 & 70,20 & 65,05 & 66,04 & 66,82 & 74,06 & 72,79 & 78,22 & 78,41 & 77,25 \\
\hline 2016 & 75,71 & 70,64 & 71,11 & 68,44 & 81,05 & 91,15 & 74,84 & 77,52 & 70,57 & 71,81 & 70,44 & 70,88 \\
\hline
\end{tabular}

A Tabela 4 apresenta os valores de previsão futura, dos 12 meses posteriores à série inicialmente apresentada, correspondendo ao ano de 2017, com o modelo $\operatorname{ARIMA}(0,1,5)$ e seus limites de confiança superior e inferior.

Tabela 4 - Previsão do preço do saco de soja a ser comercializada no estado de São Paulo, para o ano de 2017, pelo modelo $\operatorname{ARIMA}(0,1,5)$.

\begin{tabular}{cccc}
\hline Modelo & \multicolumn{3}{c}{ Preço do saco de soja de $60 \mathrm{~kg}$} \\
\cline { 2 - 4 } ARIMA $(0,1,5)$ & Previsão & LSCP* & LICP* \\
\hline jan/17 & 70,88 & 78,69 & 63,67 \\
fev/17 & 71,74 & 85,68 & 59,56 \\
mar/17 & 71,66 & 89,94 & 56,29 \\
abr/17 & 72,26 & 94,39 & 54,24 \\
mai/17 & 72,37 & 97,82 & 52,20 \\
jun/17 & 72,53 & 100,35 & 50,89 \\
jul/17 & 72,68 & 102,76 & 49,70 \\
ago/17 & 72,84 & 105,07 & 48,60 \\
set/17 & 72,99 & 107,30 & 47,59 \\
out/17 & 73,15 & 109,47 & 46,65 \\
nov/17 & 73,31 & 111,58 & 45,76 \\
dez/17 & 73,47 & 113,65 & 44,93
\end{tabular}

LSCP*: Limite Superior de Confiança da Previsão; LICP*: Limite Inferior de Confiança da Previsão.

Fonte: próprio autor.

A Figura 2 ilustra graficamente as previsões dos preços do saco de soja comercializado no estado de São Paulo, no período de 200 a 2016, bem como os limites inferior e superior de confiança da previsão, bem como as previsões mensais desses preços para o próximo ano de 2017.

Figura 2 - Valor previsto do preço da soja no estado de São Paulo usando o método ARIMA $(0,1,5)$, dentro da série de dados com os limites inferior e superior de confianças das previsões e a tendência do preço do saco de soja para o ano de 2017. 


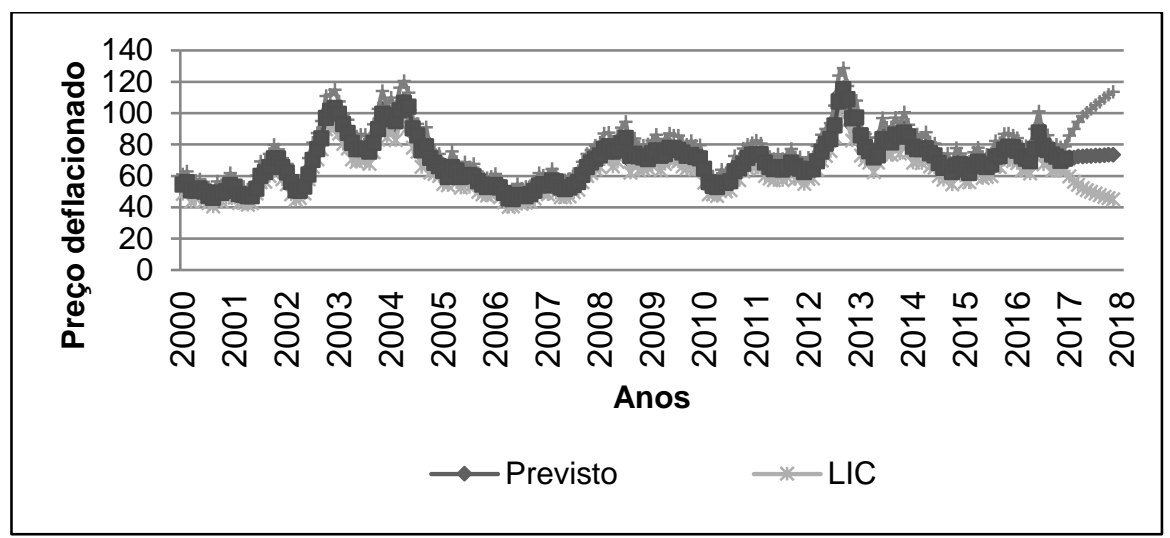

Fonte: próprio autor.

Pode-se observar, com base na Tabela 5, que os valores previstos estão próximos da média da série, e com ajuste de previsão $\left(R^{2}\right)$ de $92,4 \%$, onde pode ser confirmado com o tratamento estatístico apresentado na Tabela 6.

Tabela 5 - Estatísticas do modelo $\operatorname{ARIMA}(0,1,5)$ sobre a previsão dos preços do saco de soja de $60 \mathrm{~kg}$ no estado de São Paulo para o ano de 2017, baseado na série histórica (de 2000 a 2016).

\begin{tabular}{|c|c|c|c|c|c|c|c|c|c|}
\hline Modelo & Preditores & \multicolumn{7}{|c|}{ Estatísticas de ajuste do modelo } & Discrepantes \\
\hline $\begin{array}{c}\text { ARIMA } \\
(0,1,5) \\
\end{array}$ & $\begin{array}{c}\mathrm{N}^{\circ} \text {. de } \\
\text { Preditores }\end{array}$ & $\mathrm{R}^{2}$ & RMSE & MAPE & MAE & $\begin{array}{c}\text { BIC } \\
\text { normalizado }\end{array}$ & DF & $\begin{array}{c}p- \\
\text { valor }\end{array}$ & $\begin{array}{c}\text { №. de } \\
\text { Discrepantes }\end{array}$ \\
\hline $\begin{array}{c}\text { Preço } \\
\text { da soja }\end{array}$ & 1 & 0,924 & 4,042 & 4,314 & 3,086 & 2,846 & 16 & 0,061 & 0 \\
\hline
\end{tabular}

Fonte: próprio autor.

A partir do tratamento estatístico da Tabela 5, verificou-se que o modelo de previsão do preço do saco de soja foi altamente significativo, com $p<0,01$, e o critério de informação bayesiana ( $\mathrm{BIC}=2,846$ ), confirmou o bom desempenho da técnica preditiva adotada. As análises dos resíduos são apresentadas nas Figuras 3 e $4 \mathrm{com}$ as funções autocorrelação residual e valor residual.

Figura 3 - Gráfico dos valores residuais das funções autocorrelação total (ACF) e autocorrelação parcial (PACF) para o modelo preditivo do preço da soja utilizando a metodologia ARIMA $(0,1,5)$.

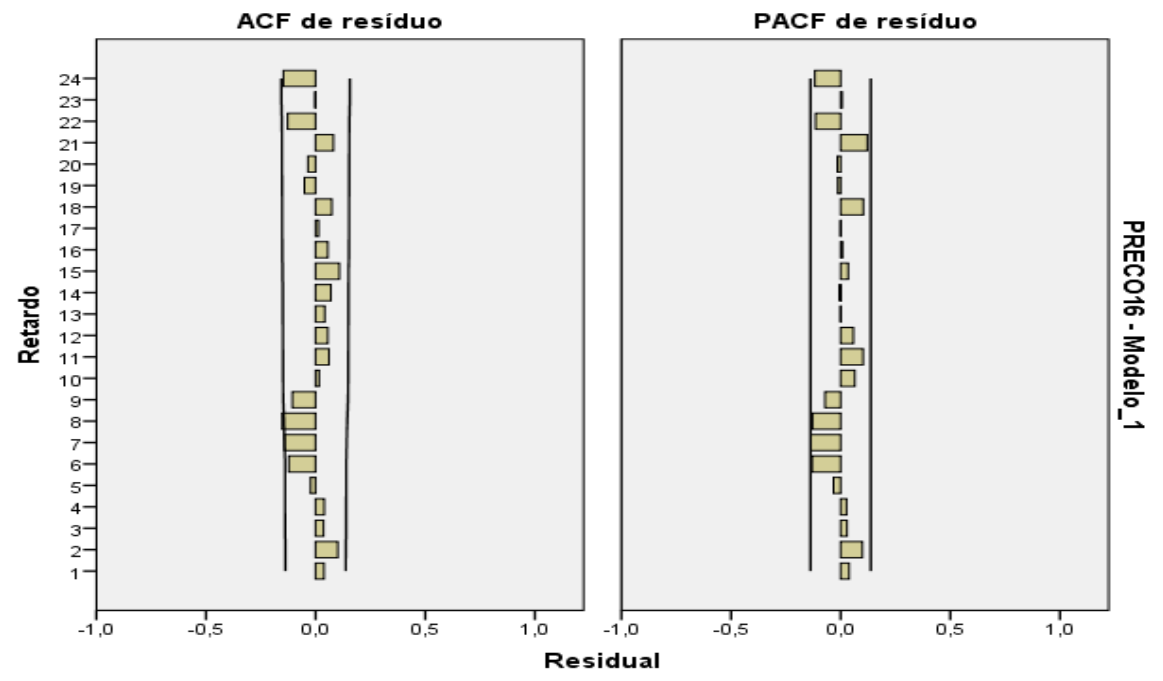


Fonte: próprio autor.

Figura 4 - Gráfico do valor residual entre os dados observados e previstos no modelo $\operatorname{ARIMA}(0,1,5)$.

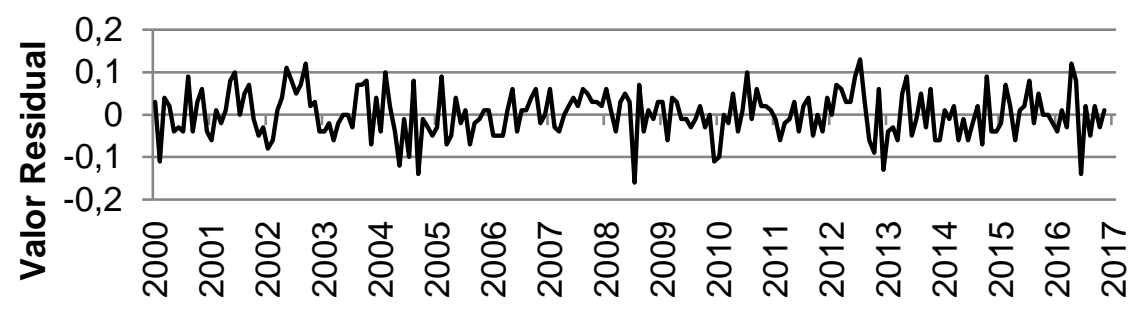

Anos

Fonte: próprio autor.

Os gráficos dos valores residuais, (Figuras 3 e 4), mostram que os valores sofrem pouca variação e se encontram bem próximos de zero, podendo ser utilizados para fazer inferências além da amostra de dados. As funções autocorrelação residual (ACF) e autocorrelação residual parcial (ACPF), confirmam que os resíduos são puramente aleatórios, critério inicial para a aplicação do método preditivo ARIMA. E por fim o gráfico da Figura 5 mostra a dispersão dos dados previstos com relação aos dados observados, em que é possível notar, a partir da distribuição dos pontos, o bom ajustamento dos dados.

Figura 5 - Gráfico de dispersão dos valores previstos com relação aos valores observados do preço da soja para toda a série temporal em análise. 


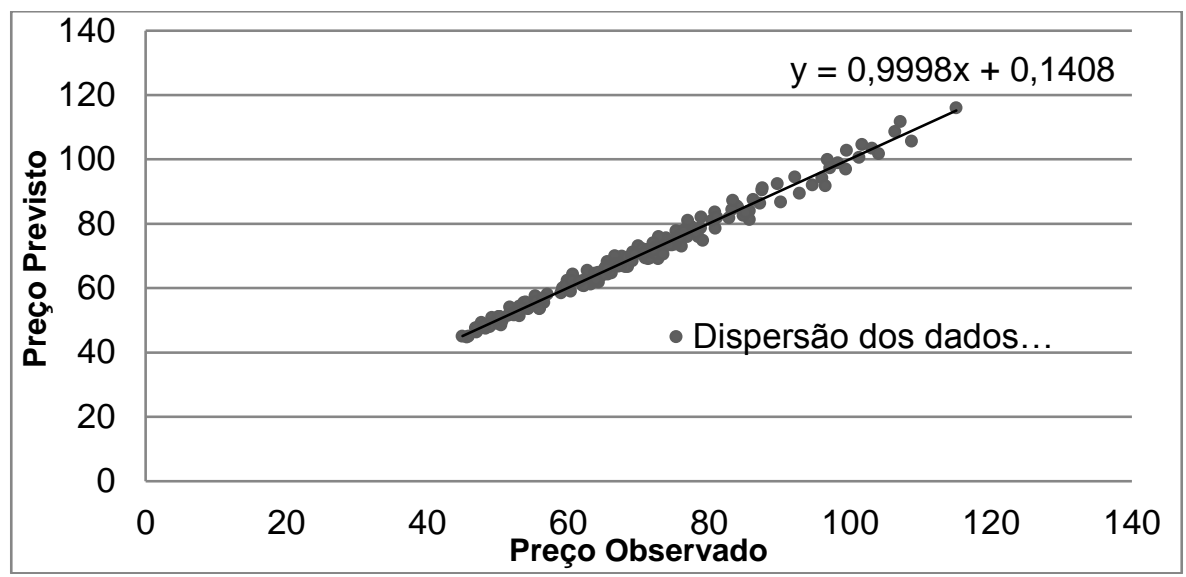

Fonte: próprio autor.

O gráfico da Figura 5 mostra que as variáveis observadas e previstas estão linearmente relacionadas, o que pode ser visualizado na equação da reta de ajuste para os dados, o que justifica o alto valor do para $R^{2}$ ao se utilizar o método ARIMA na previsão dos preços.

\section{CONCLUSÕES}

Em um cenário típico da economia onde temos: produto, produção e preço atrelado a fatores temporais oriundos dos efeitos climáticos em determinado período, faz com que todo e qualquer produto, principalmente, aqueles advindos da agricultura como a soja, que possui uma grande participação na economia nacional e internacional venha influenciar o mercado e na relação entre produtores e compradores do grão.

Após analises realizadas a respeito dos efeitos da variação estacional do preço do saco de soja comercializado no período de janeiro de 2000 a dezembro de 2016 no estado de São Paulo, pode-se verificar que em alguns meses durante o ano o preço desta cultivar tende a baixar devido a oferta disponibilizada ao grande volume da produção naquele período e aumentar de preço quando da falta do produto no mercado, o que mostra uma perfeita sazonalidade.

Existem períodos em que os preços se mostram vantajosos ao produtor para a venda desse produto, inclusive com chances de ganhos extras, não correndo tanto risco de sofrer algum prejuízo.

A técnica ARIMA foi aplicada para realizar a previsão dentro da série histórica e para os 12 meses seguintes (ano de 2017), em que se mostrou eficaz para a realização preditora dos preços do saco de soja, com coeficiente de ajuste de $92,4 \%$ e desempenho geral de 95,96\%.

Fica constatado nesta pesquisa que o momento mais vantajoso ao produtor deste grão é durante a escassez e dificuldade dos compradores na aquisição deste produto, que é justamente no período da entressafra.

Agradecimentos à Fundação Nacional de Desenvolvimento do Ensino Superior Particular - FUNADESP pela concessão de bolsa de Iniciação Científica ao aluno Luiz Henrique Brito Lemes. 


\section{REFERÊNCIAS}

AMARO, A. A.; CARVAlHO, M. A. de.; MELLO, N. T. C. de.; SILVA, V. da. Prognóstico agrícola 2005/06. Disponível em: <http://www.iea.sp.gov.br/out/verTexto.php?codTexto=3700>. Acessado em: $18 \mathrm{dez}$ 2016.

ARIAS, E. R. A., SOUZA, C. C. de, FLORES, J. M., PEREIRA, M. M. Estacionalidade dos preços e da quantidade comercializada de milho verde no mercado atacadista do CEASA de Campo Grande, estado do Mato Grosso do Sul. Informações Econômicas, v. 39, n. 3, mar. 2009.

BOTTINI, P. R.; TSUNECHIRO, A.; COSTA, F. A. G. da. Viabilidade da produção de milho verde na "safrinha". Informações Econômicas, v. 25, n. 3, p. 49-53, 1995.

CONAB. COMPANHIA NACIONAL DE ABASTECIMENTO. Séries históricas: 2016. Disponível em: <http://www.conab.gov.br/>. Acessado em: 16 dez 2016.

CAMARGO FILHO, W. P. de; MAZZEI, A. R. Mercado de verduras: planejamento e estratégia na comercialização. Informações Econômicas, v. 31, n. 3, mar. 2001.

CRUZ, J. C.; PEREIRA FILHO, I. A.; RODRIGUES, J. A. S.; FERREIRA, J. J. (Eds.). Produção e utilização de silagem de milho e sorgo. Sete Lagoas: Embrapa Milho e Sorgo, 2001. 544p.

FREITAS, M. de C. M. A cultura da soja no Brasil: o crescimento da produção brasileira e o surgimento de uma nova fronteira agrícola. Enciclopédia Biosfera, v. 7, n. 12, p. $1,2011$.

FÜRSTENAU, V. A safra de grãos de verão 2003/04 no Brasil e no Rio Grande do Sul. Indicadores Econômicos, v. 32, n. 2, p. 1-9, 2004.

GUJARATI, D. Econometria básica, 4. ed. Rio de Janeiro: Campus, 2006, 921p.

IEA. INSTITUTO DE ECONOMIA AGRÍCOLA. Soja plantada no Brasil e no Estado de São Paulo. 2016. Disponível em: <http://www.iea.agricultura.sp.gov.br>. Acessado em: 16 dez 2016.

IBGE. INSTITUTO BRASILEIRO DE GEOGRAFIA E ESTATÍSTICA. Principais Produtores Nacionais da Cultivar Soja. 2016. Disponível em: <http://www.ibge.gov.br/>. Acessado em: 20 dez 2016.

JACOBS, W.; COSTA, M. Uma referência para a construção de modelos ARIMA para previsão de demanda com base na metodologia de Box-Jenkins. In: ENCONTRO NACIONAL DE ENGENHARIA DE PRODUÇÃO (ENEGEP), 2011, Belo Horizonte MG. Anais... Belo Horizonte, Minas Gerais, 2011. 14 p.

LOVADINE, D.; BACCHI, M. R. P. Causalidade e Transmissão de Preços entre mercado interno e internacional para produtos do complexo soja. Congresso SOBER. Anais... Ribeirão Preto, SP, 2005. 
MORETTIN, P. A.; TOLOI, C. M. C. Análise de séries temporais, 2. ed. São Paulo: Edgard Blucher, 2006, 531p.

PAULA, S. R. L. de; FAVERET FILHO, P. de S. C. Panorama do complexo soja. BNDES Setorial. Setembro de 1998. Disponível em: <http://www.bndes.gov.br/SiteBNDES/export/sites/default/bndes_pt/Galerias/Arquivo s/conhecimento/bnset/set804.pdf>. Acessado em $20 \mathrm{dez} 2016$.

STEFANELO, E. L. Soja: $2008 . \quad$ Disponível em: <http://www.conab.gov.br/OlalaCMS/uploads/arquivos/10_10_25_11_12_56_soja_m aio_2008.pdf>. Acessado em: $18 \mathrm{dez} 2016$.

TSUNECHIRO, A. Valor da produção agropecuária do Brasil em 2002. Revista Informações Econômicas, v. 34, n. 2, fev. 2004. 